\title{
Taxa metabólica basal em universitários: comparação entre valores medidos e preditos
}

\author{
Basal metabolic rate of university students: \\ comparison between measured \\ and predicted values
}

Bruna Camargo BRUNETTO'

Dartagnan Pinto GUEDES ${ }^{1}$

Antonio Fernando BRUNETTO²

RE S U M O

\section{Objetivo}

Comparar a taxa metabólica basal medida por recursos de calorimetria indireta com a taxa metabólica basal estimada por equações de predição em universitários.

\section{Métodos}

A amostra foi constituída por 48 voluntários (23 moças e 25 rapazes) na faixa etária entre 20 e 25 anos. A taxa metabólica basal medida por calorimetria indireta foi comparada à taxa metabólica basal estimada pelas equações de predição propostas pela World Health Organization e por Henry \& Rees. As informações foram analisadas mediante o teste $t$ pareado, coeficiente de correlação intraclasse, desvio-padrão das diferenças e técnica de Bland-Altman.

\section{Resultados}

Não ocorreram diferenças significativas entre os valores da taxa metabólica basal medidos e estimados por ambas as equações de predição. Os coeficientes de correlação intraclasse entre os valores medidos e estimados foram significativos em linguagem estatística nos dois gêneros e mais elevados nos rapazes, e a magnitude do desvio-padrão das diferenças foi similar em ambos os gêneros. A técnica de Bland-Altman sugere comportamento de variabilidade similar entre a taxa metabólica basal medida e predita por ambas as equações, porém a taxa metabólica basal predita pela equação de Henry \& Rees apresentou coincidências mais elevadas, sobretudo entre as moças.

\section{Conclusão}

Mediante indicadores estatísticos mais conservadores, conclui-se que a taxa metabólica basal medida por recursos de calorimetria indireta e predita por ambas as equações não apresentam diferenças significativas.

\footnotetext{
${ }^{1}$ Universidade Estadual de Londrina, Centro de Educação Física e Esporte. Rod. Celso Garcia Cid (PR 445), km 380, 86051-990, Londrina, PR, Brasil. Correspondência para/Correspondence to: B.C. BRUNETTO.E-mail: <bruna_84@hotmail.com>.

2 Universidade Estadual de Londrina, Centro de Ciências da Saúde. Londrina, PR, Brasil.
} 
370 | B.C. BRUNETTO et al.

No entanto, ao considerar procedimentos estatísticos aparentemente de maior sensibilidade na detecção de eventuais diferenças entre os valores medidos e preditos, verificou-se algum comprometimento quanto à potencialidade das equações para estimar a taxa metabólica basal, apresentando viés entre 12,5\% e 15,6\% em comparação com a taxa metabólica basal medida por recursos de calorimetria indireta.

Termos de indexação: Calorimetria indireta. Estudantes. Metabólismo basal. Previsões.

\section{A B S T R A C T}

\section{Objective}

The aim of this work is to compare the basal metabolic rate determined by indirect calorimetry with the basal metabolic rate estimated by prediction equations in university students.

\section{Methods}

The sample consisted of 48 Brazilian volunteers (23 women and 25 men), aged 20-25 years. The basal metabolic rate determined by indirect calorimetry was compared with the basal metabolic rate estimated by the World Health Organization and Henry \& Rees equations. The data was analyzed by the paired t-test, intra-class correlation coefficients, standard deviation of differences and Bland and Altman technique.

\section{Results}

There were no statistically significant differences between measured and estimated basal metabolic rates by both equations. Correlation coefficients between measured and estimated values were higher in men, however statistically significant in both genders. The magnitude of the standard deviation of differences was similar in both genders. The Bland and Altman technique suggests a similar variability behavior between the measured basal metabolic rate and that estimated by both equations. However, basal metabolic rate predicted by the Henry \& Rees equation showed greater concordance than that predicted by the World Health Organization equation, especially in women.

\section{Conclusion}

Due to the use of more conservative statistical indicators, it appears that the basal metabolic rates measured by indirect calorimetry and predicted by both equations do not differ significantly. However, when statistical procedures with an apparently higher sensitivity for detecting differences between measured and predicted rates are used, the potential of the equations to estimate the basal metabolic rate was somewhat compromised. There was a bias of $12.5 \%$ to $15.6 \%$ in comparison with the basal metabolic rate determined by indirect calorimetry.

Indexing terms: Calorimetry indirect. Students. Basal metabolism. Forecasting.

\section{N T R O D U ÇÃ O}

A Taxa Metabólica Basal (TMB), definida como a quantidade básica de energia para a manutenção das funções do organismo em condições de repouso, varia em função do gênero e da idade, sendo comumente empregada como indicador associado à porção significativa (60-70\%) da quantidade total do dispêndio de energia diário ${ }^{1}$. Dessa forma, o conhecimento quanto aos valores da TMB torna-se extremamente útil para o controle do equilíbrio energético no corpo humano.

A calorimetria indireta, considerada um procedimento de elevada precisão e exatidão para a medida da TMB, é uma técnica que mede a taxa de captação de oxigênio e a taxa de produção de gás carbônico mediante as trocas respiratórias, associadas à oxidação de carboidratos, lipídeos e proteínas ${ }^{2-4}$. No entanto, o elevado custo dos equipamentos envolvidos, a sofisticação de seus procedimentos e a necessidade de pessoal especializado limitam muito sua utilização quando da necessidade de desenvolver avaliações equivalentes à TMB em ambiente clínico.

O primeiro estudo envolvendo a estimativa da TMB por intermédio de equações de predição foi realizado por Harris \& Benedict em 19195. Nesse caso, as equações foram derivadas envolvendo 
variáveis antropométricas e uma amostra representativa de homens, mulheres e crianças. Atualmente as equações propostas por Harris \& Benedict, apesar de não estarem isentas de limitações e viés de estimativa, são frequentemente empregadas para a predição da $\mathrm{TMB}^{6}$. Porém foi somente a partir da disseminação das equações sugeridas pela World Health Organization', mediante ajustes em uma compilação de dados publicados por Schofield ${ }^{7}$, que se despertou o interesse pela tentativa de validação de equações para estimativa da TMB em diferentes populações. Foi, sobretudo, pela recomendação da World Health Organization, na época, de que as necessidades energéticas diárias fossem estabelecidas em múltiplos da TMB, e não mais pela ingestão energética, como se fazia até então' ${ }^{1}$. Dessa forma, acreditava-se que a proposição e a validação de equações de predição poderiam trazer maior facilidade e praticidade na realização de estudos populacionais.

Recentemente, a World Health Organization procurou atualizar as equações propostas originalmente em 1985; contudo, embora as novas equações possam apresentar algum mérito, como é o caso de reduções na magnitude dos erros de predição e dos vieses de superestimativa entre os valores observados nos homens, foi concluído que as adaptações realizadas não são robustas o suficiente para justificar sua adoção.

Nesse sentido, estudos têm demonstrado que as equações sugeridas pela World Health Organization fornecem, na maioria dos casos, valores de estimativa da TMB mais elevados quando comparados aos valores medidos da TMB, tanto em populações pediátricas ${ }^{9,10}$ como em adultos $^{11}$, e particularmente entre os residentes nos trópi$\cos ^{12}$. Essas diferenças possivelmente possam ser explicadas pelo fato de as equações terem sido derivadas de populações europeia e norte-americana, bem como pela importante influência de alguns componentes relacionados ao gênero, à idade e, especialmente, à composição corporal, que possivelmente possam apresentar algum impacto proveniente das diferentes condições ambientais e climáticas em que vivem os indivíduos.
Considerando essas evidências, Henry \& Rees $^{12}$ propuseram uma série de equações direcionadas à predição dos valores da TMB para populações que vivem em regiões tropicais. Em geral, o uso dessas equações tem apresentado valores associados à estimativa da TMB menores do que os obtidos pela equação da World Health Organization ${ }^{13-15}$.

Tentativas de validação envolvendo recursos de calorimetria indireta direcionados à medida da TMB apontam para valores médios que superestimam por volta de $2 \%$ a $14 \%$ a TMB obtida por intermédio de equações preditivas. No entanto, analisando casos individuais, os valores preditos em uma mesma condição podem coincidir ou subestimar discretamente a TMB medida em alguns indivíduos ${ }^{16}$.

Especificamente na população brasileira, estudos têm apontado limitações quanto ao emprego das equações sugeridas para uso internacional, tanto em amostras provenientes de região temperada ${ }^{15}$ como de região tropical ${ }^{14}$ do país, fato que coloca em questão a aplicabilidade dessas equações em nossa realidade. Mesmo assim, não se pode deixar de considerar aspectos relacionados à heterogeneidade das populações dos estudos referenciais, à metodologia empregada e à variabilidade nos valores da TMB, que podem influenciar decisivamente a magnitude dos resultados.

Dessa forma, baseando-se nos pressupostos de que as equações preditivas propostas até então não têm oferecido satisfatório nível de concordância; que a variação dos vieses de estimativa é de considerável magnitude em diferentes populações; e que as intervenções em programas de controle do peso corporal são facilitadas quando o equilíbrio energético é realizado mediante a identificação dos valores da TMB ${ }^{17}$, o objetivo do presente estudo foi comparar as medidas da TMB obtidas mediante recursos de calorimetria indireta com medidas estimadas por intermédio das equações preconizadas pela World Health Organization e pelo Henry \& Rees ${ }^{12}$ em uma amostra de universitários, na tentativa de apontar novos direcionamentos para a predição da TMB. 


\section{MÉ TO D OS}

A amostra do estudo foi constituída por 48 universitários (23 moças e 25 rapazes) de diferentes cursos da Universidade Estadual de Londrina (PR) na faixa etária de 20 a 25 anos. A participação no estudo foi de caráter voluntário e como critérios de exclusão foram considerados: (a) desistência anterior à realização das medidas; (b) não adesão ao protocolo de estudo; (c) utilização de algum medicamento que pudesse modificar as variáveis de estudo; e/ou (d) intolerância ao protocolo de medida por questões de desconforto e de não adaptação ao equipamento de calorimetria indireta.

Para a determinação dos indicadores antropométricos foram utilizadas medidas de peso corporal e estatura, realizadas previamente ao início da coleta de gases. Para as medidas de estatura, foi empregado estadiômetro de madeira com definição de $0,1 \mathrm{~cm}$, e para as medidas de peso corporal, balança antropométrica eletrônica marca Filizola com definição de 0,1 kg.

Para as medidas da TMB foi utilizada a técnica de calorimetria indireta, mediante o aparelho portátil VO2000 - Medical Graphics, que consiste de máscara facial conectada ao medidor de ventilação e ao analisador de gases e oclusor nasal. Antes do início de cada medida, o equipamento foi calibrado com concentrações conhecidas de gases $\left(17 \% \mathrm{O}_{2}\right.$ e $\left.5 \% \mathrm{CO}_{2}\right)$. Dados da captação de oxigênio e da produção de gás carbônico foram convertidos em dispêndio energético, utilizando-se a equação $\left[3,9\left(\mathrm{VO}_{2}\right)+1,1\left(\mathrm{VCO}_{2}\right)\right]$, descrita por Weir ${ }^{18}$.

O protocolo foi aplicado no período matutino, entre sete e nove horas da manhã, sob condições de 12 horas de jejum, oito horas de sono e ausência de esforços físicos intensos no dia anterior. O procedimento consistiu de 10 minutos de repouso absoluto, seguidos de 20 minutos de coleta e análise dos gases com os indivíduos deitados na posição supino em condições similares para todos os participantes da amostra, na tentativa de minimizar eventual variabilidade aleatória dos resul- tados. Durante a coleta, os sujeitos foram adequadamente orientados para que permanecessem em repouso absoluto. As medidas dos gases foram coletadas em um tempo fixo a cada 10 segundos durante os 20 minutos. $O$ experimento foi realizado em sala com condições adequadas para a medida da TMB: temperatura ambiente controlada entre 22 e $24^{\circ} \mathrm{C}$, baixa luminosidade e sem ruídos.

As equações de predição da TMB utilizadas para comparação foram:

$$
\begin{aligned}
& \text { World Health Organization }{ }^{1} \\
& \text { Moças - }[(0,0615 \times \text { massa corporal })+2,08] \\
& \text { Rapazes - }[(0,0640 \times \text { massa corporal })+2,84] \\
& \text { Henry \& Rees }{ }^{12}
\end{aligned}
$$$$
\text { Moças - }[(0,048 \times \text { massa corporal })+2,562)]
$$$$
\text { Rapazes - }[(0,056 \times \text { massa corporal }+2,800]
$$

Originalmente, os valores preditos pelas equações são apresentados em unidades de medida quilojoule. Dessa forma, para a conversão em unidades de medida quilocaloria, os resultados foram multiplicados por 239.

Para análise das informações coletadas, foi utilizado o pacote computadorizado Statistical Package for the Social Science (SPSS), versão 17.0. A caracterização da amostra foi realizada mediante procedimentos da estatística descritiva (Média - M, Desvio-Padrão - DP). Recursos relacionados ao teste $t$ pareado, ao coeficiente de correlação intraclasse e ao desvio-padrão das diferenças foram empregados para análise das informações associadas aos valores medidos e preditos pelas equações da TMB. Foram empregados, também, plotagens em diagramas de dispersão de acordo com a técnica desenvolvida por Bland \& Altman ${ }^{19}$ para análise das coincidências entre os valores medidos e preditos da TMB. Todas as análises utilizaram nível de significância de $p<0,05$.

Os procedimentos empregados no estudo foram aprovados pelo Comitê de Ética em Pesquisa da Universidade Estadual de Londrina (Parecer CEP 081/2007). 


\section{RESULTADOS}

Indicadores estatísticos quanto à comparação das medidas da TMB obtidas mediante recursos de calorimetria indireta e preditas por intermédio das equações são disponibilizadas na Tabela 1. Os resultados mostram que não ocorreram diferenças significativas entre os valores medidos e os valores preditos por ambas as equações (Tabela 2). Os coeficientes de correlação intraclasse entre os valores medidos e preditos foram mais elevados nos rapazes, porém significativos em linguagem estatística nos dois gêneros. Os valores equivalentes ao desvio-padrão das diferenças foram similares em ambos os gêneros (Tabela 1).

Os diagramas de dispersão com a plotagem dos valores médios medidos e preditos da TMB (abscissa) e as diferenças individuais entre os valores medidos e preditos pelas duas equações (ordenada) são procedimento de análise proposto por Bland e Altman ${ }^{19}$ que permite visualizar as diferenças médias e os limites extremos de concor- dância (2 DP da diferença) entre os valores medidos e preditos da TMB. A disposição gráfica dos dados sugere comportamento de variabilidade similar entre as moças e os rapazes, e entre os valores medidos e preditos da TMB por ambas as equações. Porém os valores da TMB preditos pela equação proposta por Henry \& Rees ${ }^{12}$ apresentaram coincidências mais elevadas, sobretudo entre as moças (Figura 1).

\section{I S C U S S Ã O}

No presente estudo, a TMB não apresentou diferenças significativas entre os valores medidos e preditos por ambas as equações consideradas. Mesmo assim, a equação da World Health Organization apresentou, em média, valores preditos com diferença de 15,6\% (211 kcal/dia) para as moças e de $13,1 \%$ (225kcal/dia) para os rapazes em comparação com os valores medidos por calorimetria indireta, enquanto a equação de Henry \& Rees ${ }^{12}$ apresentou diferença média de 14,1\%

Tabela 1. Comparações entre os valores da taxa metabólica basal medida por intermédio da técnica de calorimetria indireta e por equações preditivas em universitários de diferentes cursos da Universidade Estadual de Londrina. Londrina (PR), 2007.

\begin{tabular}{lccc}
\hline Sexo & Teste $t$ & Correlação intraclasse & Desvio-padrão das diferenças \\
\hline Moças & & & \\
Equação $_{\text {FAONHO/UNU-1985 }}$ & $1,840(p=0,0725)$ & $0,456(p=0,020)$ & $211 \mathrm{kcal}$ \\
Equação $_{\text {Henry \& Rees }}{ }^{2}$ & $0,498(p=0,6212)$ & $0,467(p=0,020)$ & $191 \mathrm{kcal}$ \\
Rapazes & $1,329(p=0,1903)$ & $0,649(p=0,000)$ & $225 \mathrm{kcal}$ \\
Equação $_{\text {FAONHO/UNU-1985 }}$ & $1,022(p=0,3117)$ & $0,651(p=0,000)$ & $215 \mathrm{kcal}$ \\
Equação $_{\text {Henry \& Rees12 }}$ & & & \\
\hline
\end{tabular}

Tabela 2. Estatística descritiva de variáveis analisadas em estudo envolvendo universitários de diferentes cursos da Universidade Estadual de Londrina. Londrina (PR), 2007

\begin{tabular}{|c|c|c|c|c|c|}
\hline & \multicolumn{2}{|c|}{ Moças $(n=23)$} & \multicolumn{2}{|c|}{ Rapazes $(n=25)$} & \multirow{2}{*}{ Teste $t$} \\
\hline & M & DP & M & DP & \\
\hline Peso corporal (kg) & 56,31 & 7,48 & 72,10 & 9,83 & $p<0,001$ \\
\hline Estatura $(\mathrm{cm})$ & 164,29 & 7,46 & 177,86 & 7,88 & $p<0,001$ \\
\hline $\mathrm{IMC}\left(\mathrm{kg} / \mathrm{m}^{2}\right)$ & 20,80 & 1,79 & 22,82 & 3,06 & $p<0,008$ \\
\hline TMB ${ }_{\text {medida }}(\mathrm{kcal} / \mathrm{dia})$ & 1349,39 & 221,35 & 1716,76 & 281,79 & $p<0,001$ \\
\hline $\mathrm{TMB}_{\text {FAONHO/UNU }}(\mathrm{kcal} / \mathrm{dia})$ & 1258,29 & 85,80 & 1634,13 & 131,53 & $p<0,001$ \\
\hline TMB ${ }_{\text {Henry \& Rees } 12}(\mathrm{kcal} / \mathrm{dia})$ & 1323,74 & 109,94 & 1782,07 & 150,36 & $p<0,001$ \\
\hline
\end{tabular}

M: média; DP: desvio-padrão; IMC: índice de massa corporal; TMB: taxa metabólica basal. 
$\mathrm{FAO} / \mathrm{WHO} / \mathrm{UNU}^{\prime}$


Henry \& Rees ${ }^{12}$
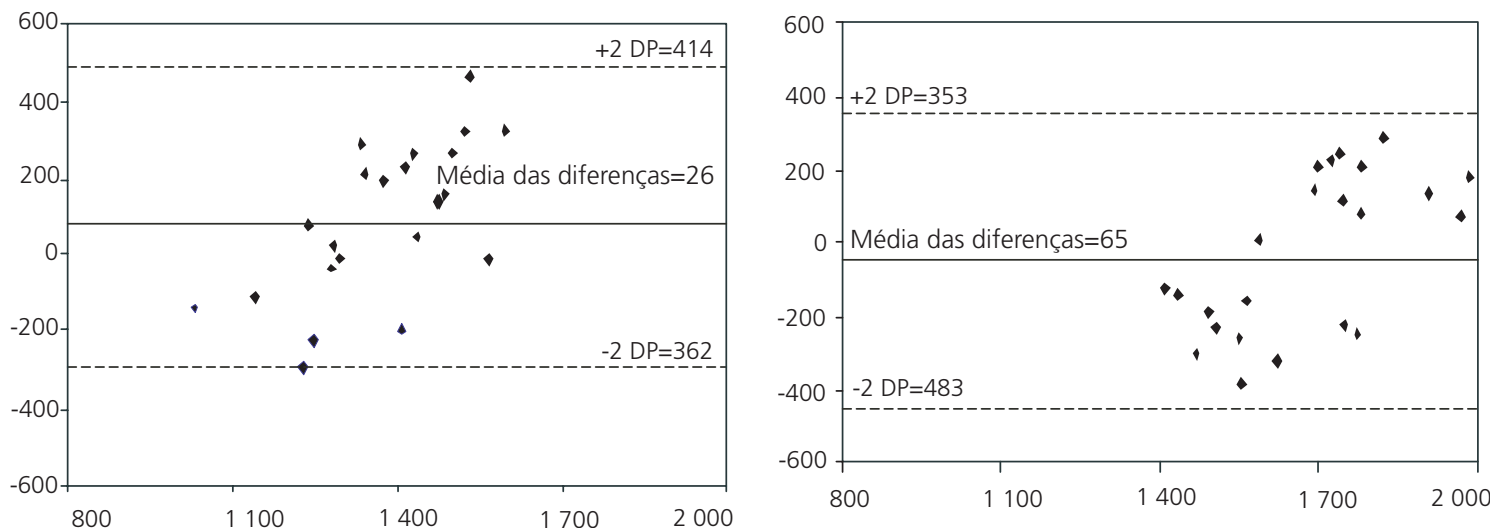

Figura 1. Plotagem de Bland-Altman para os limites de concordância entre os valores medidos e preditos da taxa metabólica basal pelas equações FAO/WHO/UNU ${ }^{1}$ e Henry \& Rees ${ }^{12}$ em universitários de diferentes cursos da Universidade Estadual de Londrina. Londrina (PR), 2007.

(191kcal/dia) para as moças e de $12,5 \%$ (215kcal/ dia) para os rapazes. Essas diferenças se mostraram superiores às encontradas no estudo de Cruz et al. ${ }^{14}$, que, ao avaliarem mulheres universitárias, encontraram diferenças de $12,5 \%$ quando da comparação dos valores da TMB medida por calorimetria indireta e da TMB predita pela equação recomendada pela World Health Organization', e diferenças de 7,2\% quando da comparação dos valores da TMB medida e a TMB predita pela equação sugerida por Henry \& Rees ${ }^{12}$.

Resultados similares foram encontrados nos estudos de Wahrlich \& Anjos $^{15}$. Chama a atenção o fato de Wahrlich \& Anjos $^{15}$ terem realizado seus estudos envolvendo amostras da cidade de Porto
Alegre (RS), região considerada temperada, e encontrado viés de estimativas pelas equações de Henry \& Rees ${ }^{12}$ similares aos de Cruz et al. ${ }^{14}$, que envolveram sujeitos residentes em região tropical (Rio de Janeiro). A expectativa, no entanto, era de que as estimativas se mostrassem adequadas quando da utilização das equações da World Health Organization, uma vez que essas foram derivadas em populações de zonas temperadas. Outros fatores, além do clima, podem ter influenciado esses resultados, considerando a grande variabilidade de condições ambientais e de vida nas diversas regiões do país.

No presente estudo, apesar de as comparações entre a TMB medida por calorimetria indire- 
ta e a TMB predita pelas equações apontarem diferenças associadas à TMB predita pela equação de Henry \& Rees de menor magnitude que pela equação da World Health Organization, esperava-se que essas diferenças fossem ainda menores por utilizar uma equação destinada a populações vivendo nos trópicos. No estudo original, quando da comparação entre os valores da TMB predita pelas equações ajustadas por Henry \& Rees e pelas equações da World Health Organization, foram encontradas diferenças significativas em torno de $9 \%$ menores entre os homens e $5,4 \%$ entre as mulheres. Uma possível explicação para esses achados é o fato de a amostra selecionada no presente estudo, aparentemente homogênea com relação às características morfológicas, ter apresentado uma variabilidade excessivamente elevada entre os valores medidos da TMB (DP de $221,35 \mathrm{kcal} /$ dia nas moças e $281,79 \mathrm{kcal} / \mathrm{dia}$ nos rapazes). Essa maior variabilidade, por sua vez, pode ter sido ocasionada pelos fatores biológicos característicos da amostra e pelo método e equipamento empregado na coleta dos dados de calorimetria indireta.

Lawrence et al. ${ }^{20}$ não encontraram diferenças significativas na TMB medida por calorimetria indireta de mulheres entre 20 e 30 anos da Escócia, Gâmbia e Tailândia com composição corporal similar. No entanto, ao utilizar a equação da World Health Organization, os resultados de seus estudos apontaram valores que subestimam em torno de 5 a $10 \%$ a TMB das mulheres de Gâmbia e Tailândia, e elevada coincidência entre os valores medidos e preditos da TMB das mulheres escocesas. Da mesma forma, Soares et al. ${ }^{21}$, em uma amostra de homens, ao desenvolverem equações especificas para a população da Índia, não encontraram diferenças significativas ao utilizá-las na população norte-americana. Porém, ao utilizarem essas mesmas equações em populações de países europeus, a TMB se mostrou superestimada em 6,3\%. Estudos de Nhung et al. ${ }^{22}$, também envolvendo amostras de sujeitos que vivem em região tropical (Vietnã), na tentativa de delinear equações específicas, sugerem valores de TMB predita pelas suas equações 7,4\% e 9,0\% menores que valores preditos pelas equações preconizadas pela World Health Organization para homens e mulheres respectivamente. Considerando os estudos mencionados, parece não ser conclusivo o impacto do clima e da etnia na estimativa por equações preditivas dos valores associados à TMB de diferentes populações.

Estudos desenvolvidos por Müller et al. ${ }^{23}$, ao reanalisarem o banco de dados que originou as equações sugeridas pela World Health Organization, colocam em questão sua utilização nas sociedades modernas, considerando que, desde sua recomendação para uso internacional em 1985, a população mundial se tornou mais obesa, menos ativa fisicamente e modificou acentuadamente seus hábitos alimentares especialmente pela inserção dos avanços tecnológicos no cotidiano, o que contribui para possíveis alterações na composição corporal e, por consequência, no equilíbrio energético diário. Mesmo assim, acreditamos que uma das justificativas para o uso das equações da World Health Organization, apesar de suas limitações, seja o fato de os estudos de proposição de novas equações se restringirem a amostras de pequeno tamanho e se limitarem a características bastante específicas.

De fato, são grandes as dificuldades no desenvolvimento de estudos epidemiológicos nos dias atuais, especialmente pelas constantes modificações nas características das populações. Essa situação tem contribuído para que os estudos comparativos sejam realizados em segmentos específicos da população. No entanto, na realização de estudos de caráter comparativo, um aspecto que preocupa é a ausência de consenso quanto à padronização de métodos destinados à medida da TMB. Recentemente, estudos de Wahrlich et al. ${ }^{24}$ procuraram validar a utilização do aparelho de calorimetria indireta VO2000, equipamento também utilizado no presente estudo, diante do calorímetro de referência Deltatrac ${ }^{\mathrm{TM}}$. Apesar de serem encontrados indicadores de validação satisfatórios, os autores ressaltam a ocorrência de casos de hiperventilação e de não adaptação à máscara facial, ocasionando desconforto em 
alguns sujeitos. Situação similar foi observada no presente estudo, no entanto não houve controle da frequência desses acontecimentos, impedindo, portanto, a obtenção de informações com relação ao grau de comprometimento desses fenômenos nos valores da TMB medida.

No que se refere aos procedimentos metodológicos, o tempo de coleta dos gases e o critério de seleção dos dados para o cálculo da TMB se mostram bastante diferentes entre os estudos. No presente estudo, a coleta de gases foi realizada por 20 minutos, sendo utilizados todos os dados para o cálculo da TMB. No entanto, alguns estudos utilizaram protocolos com coleta de gases por 15-25 minutos, não considerando os 5 minutos iniciais para efeito de cálculo ${ }^{14,15}$. Outros estudos coletaram dados por 60 minutos, desconsiderando os 20 minutos iniciais ${ }^{25}$ ou selecionando o período de tempo de maior estabilidade dos dados, resultando em um tempo de coleta maior ou menor de acordo com a variabilidade observada ${ }^{26}$. Assumindo que a variabilidade da medida da TMB possa ser estabelecida por fatores biológicos, instrumentos de medida e viés de medida, e que os viéses de medida incluem o erro técnico do avaliador e as variações quanto à adaptação dos avaliados aos equipamentos de medida ${ }^{17}$, parece razoável assumir que esses fatores possam explicar a acentuada variabilidade dos valores individuais encontrados no presente estudo. Não obstante, quantificar a contribuição desses vieses na medida da TMB torna-se uma tarefa difícil visto a subjetividade desses fatores.

Diante dos resultados encontrados no presente estudo, pode-se inferir que indicadores estatísticos mais conservadores sugerem que a TMB medida por recursos de calorimetria indireta e predita pelas equações recomendadas pela World Health Organization ${ }^{1}$ e pelo Henry \& Rees ${ }^{12}$ não apresentam diferenças significativas. No entanto, ao considerar procedimentos estatísticos aparentemente de maior sensibilidade na detecção de eventuais diferenças individuais entre os valores medidos e preditos, verifica-se algum comprometimento quanto à potencialidade de ambas as equações para estimar a TMB, apresentando viés entre $12,5 \%$ e $15,6 \%$ em comparação com a TMB medida por recursos de calorimetria indireta.

A inadequação das equações de predição pode comprometer o estabelecimento de requerimentos energéticos adequados nos diferentes segmentos da população, induzindo eventualmente a equívocos nas tomadas de decisões em programas de controle do peso corporal. Dessa forma, faz-se necessário o estabelecimento de melhores entendimentos acerca dos determinantes da TMB na tentativa de obter valores preditos da TMB mais próximos dos valores reais, na perspectiva de definir alternativas apropriadas para o estabelecimento do equilíbrio energético e a manutenção de um estado apropriado de saúde da população.

\section{OLABORADORES}

B.C. BRUNETTO participou da coleta de dados e da redação do artigo. D.P. GUEDES participou da análise dos dados e da correção final do artigo. A.F. BRUNETTO participou da redação do artigo.

\section{REFERÊ N CIAS}

1. World Health Organization. Energy and protein requirements. Geneva: WHO; 1985. WHO Technical Report Series, 724.

2. Ferrannini $\mathrm{E}$. The theoretical bases of indirect calorimetry: a review. Metabolism. 1988; 37(3): 287-301.

3. Murgatroyd PR, Shetty PS, Prentice AM. Techniques for the measurement of human energy expenditure: a practical guide. Int J Obes Relat Metab Disord. 1993; 17(10):549-68.

4. Green JH. Assessment of energy requirements. In: Heatley RV, Green JH, Losowsky MS. Consensus in clinical nutrition. Cambridge: University Press; 1994.

5. Harris J, Benedict G. A biometric study of basal metabolism in man. Washington (DC): Carnegie Institution; 1919. Carnegie Institution of Washington Publication, 279.

6. Frankenfield DC, Muth ER, Rowe WA. The HarrisBenedict studies of human basal metabolism: 
history and limitations. J Am Diet Assoc. 1998; 98(4):439-45.

7. Schofield WN. Predicting basal metabolic rate, new standards and review of previous work. Hum Nutr Clin Nutr. 1985; 39(Suppl.1):5-41.

8. World Health Organization. Human energy requirements: food and nutrition. Rome: WHO; 2001. Technical Report Series 1.

9. Rodriguez G, Moreno LA, Sarria A, Fleta J, Bueno $M$. Resting energy expenditure in children and adolescents: agreement between calorimetry and prediction equations. Clin Nutr. 2002; 21(3): 255-60.

10. Dietz WH, Bandini LG, Schoeller DA. Estimates of metabolic rate in obese and non obese adolescents. J Pediatr. 1991; 118(1):146-9.

11. Frankenfield DC, Rowe WA, Smith JS, Cooney RN. Validation of several established equations for resting metabolic rate in obese and nonobese people. J Am Diet Assoc. 2003; 103(9):1152-9.

12. Henry CJK, Rees DG. New predictive equations for the estimation of basal metabolic rate in tropical peoples. Eur J Clin Nutr. 1991; 45(4):177-85.

13. Piers LS, Shetty OS. Basal metabolic rates of Indian women. Eur J Clin Nutr. 1993; 47(8):586-91.

14. Cruz CM, Silva AF, Anjos LA. A taxa metabólica basal é superestimada pelas equações preditivas em universitárias do Rio de Janeiro, Brasil. Arch Latinoam Nutr. 1999; 49(3):232-7.

15. Wahrlich $V$, Anjos LA. Validação de equações de predição da taxa metabólica basal em mulheres residentes em Porto Alegre, RS, Brasil. Rev Saúde Pública. 2001; 35(1):39-45.

16. Wahrlich V, Anjos LA. Aspectos históricos e metodológicos da medição e estimativa da taxa metabólica basal: uma revisão de literatura. Cad Saúde Pública. 2001; 17(4):801-17.

17. Haugen HA, Melanson EL, Tran ZV, Kearney JT, Hill JO. Variability of measured resting metabolic rate. Am J Clin Nutr. 2003; 78(6):1141-5.

18. Weir JB. New methods for calculating metabolic rate with special reference to protein metabolism. J Physiol. 1949; 5(2):99-114.
19. Bland JM, Altman DG. Statistical methods for assessing agreement between two methods for clinical measurement. Lancet. 1986; 1(8476): 307-10.

20. Lawrence M, Thongprasert K, Durnin JVGA. Between-group differences in basal metabolic rate: an analysis of data collected in Scotland, the Gambia and Thailand. Eur J Clin Nutr. 1988; 42(10): 877-91.

21. Soares MJ, Francis DG, Shetty PS. Predictive equations for basal metabolic rates of Indian males. Eur J Clin Nutr. 1993; 47(6):389-94.

22. Nhung BT, Khan NC, Hop LT, Lien DT, Le DS, Hien $V T$, et al. FAOMHO/UNU equations overestimate resting metabolic rate in Vietnamese adults. Eur J Clin Nutr. 2005; 59(10):1099-104.

23. Müller MJ, Westphal $A B$, Klaus S, Kreymann G, Lührmann PM, Berthold M, et al. World Health Organization equations have shortcomings for predicting resting energy expenditure in persons from a modern, affluent population: generation of a new reference standard from a retrospective analysis of a German database of resting energy expenditure. Am J Clin Nutr. 2004; 80(5): 1379-90.

24. Wahrlich V, Anjos LA, Going SB, Lohman TG. Validation of the VO2000 calorimeter for measuring resting metabolic rate. Clin Nutr. 2006; 25(4):687-92.

25. Jones A, Shen W, ST-Onge MP, Gallagher D, Heshka $S$, Wang $Z$, et al. Body-composition differences between African American and white women: relation to resting energy requirements. Am J Clin Nutr. 2004; 79(5):780-6.

26. Schneider P, Meyer F. As equações de predição da taxa metabólica basal são apropriadas para adolescentes com sobrepeso e obesidade? Rev Bras Med Esporte. 2005; 11(3):193-6.

Recebido em: 16/4/2007

Versão final reapresentada em: 3/12/2009 Aprovado em: 16/3/2010 